\title{
Medical Aftercare of Sexually Assaulted Victims by Forensic Physician
}

\section{Mohammed Madadin*}

Forensic Medicine Center in Dammam, Kingdom of Saudi Arabia, Saudi Arabia

\begin{abstract}
Victims of sexual assault must be completely evaluated and managed, while the history, physical examination and forensic evidence collection is almost standard procedures, many of forensic physicians in different countries vary in medical aftercare of the victim. Aftercare is a vital component of recovery for the sexual assault victim, every effort must be made to provide the victim with adequate care among rape victims, and follow-up rates are low in many countries, especially developing countries like Saudi Arabia and Egypt. Many studies showed those who do come in for follow-up have physical complaints and health-related concerns that are related to their assault. This review is designed to assist states in the development of sexual assault protocols especially in medical after care. The issues were addressed in when and how medical aftercare provided as in developed countries such as UK and USA. Such care must be provided and evaluated by forensic physician then refer to another medical discipline if needed, the components of this aftercare include HIV, Hepatitis B, STD prophylaxis and screening in addition to pregnancy control and psychological support .Because it is difficult to evaluate victims who did not undergo appropriate post-assault follow up, it is good to look for victim who undergo under appropriate follow up and see how much they benefit and passed the risk of physical and psychological harm.
\end{abstract}

\section{Advantages of Medical Aftercare of Sexually Assaulted Victims by Forensic Physician Instead of Referral}

As part of forensic doctor job, the forensic physician must consider the risk that the victims may have acquired as a result of the alleged sexual assault. It is essential for the forensic physician to conduct appropriate risk assessment for STI, Hepatitis B, HIV , illegal pregnancy and psychological harm.

We hypothesized that medical aftercare performed by the forensic physician may be associated with more benefits for both the victim and the legal component based on the following facts:

- Examinations performed by the forensic physician may be associated with higher rates of follow-up than examinations conducted by obstetrics and gynecology residents or other residents because forensic doctors have more face-to-face time with the patients and more experience with sexual assault examinations.

- Forensic Examiner can consider and conduct risk assessment for possible medical needs with immediate intervention; Time factor for the intervention for the medical needs is extremely important and life-saving.

- Medical aftercare performed by forensic doctor may ensure proper and easier maintenance of chain of custody.

- Like all infections, there will be an incubation period between exposure to pathogen and infection. Screening at the time of initial examination is not enough and from medico- legal point of view, forensic physician must inform the victim the importance of follow-up for detecting and treating any infections that could link alleged offender to complainant.

- Medical aftercare may be better to be done by the forensic medical examiner because he can interpretate all results within the context of the entire case investigations depending on his qualification and experiment.

- Avoidance of delay in obtaining the results and apart appointments as in case of referral.
- Some patients prefer to attend the same place and same doctor.

- Some injuries sustained during the assault my not yet appeared at the time of initial examination like the bruises.

- Differentiation between natural diseases like skin diseases and assault induced injuries is critical point for the forensic physician.

So, follow-up of these cases and examination at a later stage by forensic doctor is of great help to decide whether these are actual injuries or not.

\section{Emergent Contraception}

Giving of emergent contraception to victim by forensic physician is great important as time factor is critical and Indications Depend on the Nature of the assault, Time from assault to examination, menstrual status, and Current method of contraception [1].

Currently, there are 2 main methods of emergency contraception Hormonal and Intra-uterine device (IUD), The Hormonal such as Levonorgestrel (Levonelle) which should be given as single dose 1.5 mg soon as possible after unprotected sexual intercourse (UPSI) and within 72 hours. The other hormonal contraception Ulipristal acetate (ellaOne) should be given as soon as possible within 120 hours [2].

Should be fitted the other group which is Intra-uterine device (IUD) should be fitted within 5 days of UPSI [3].

Efficacy of Levonorgestre if taken within 24 hours will be $95 \% \mathrm{w}$

*Corresponding author: Mohammed Madadin, Forensic Medicine Cente in Dammam, Kingdom of Saudi Arabia, Saudi Arabia, E-mail: dr_msm83@ hotmail.com

Received March 17, 2012; Accepted June 22, 2012; Published June 24, 2012

Citation: Madadin M (2012) Medical Aftercare of Sexually Assaulted Victims by Forensic Physician. J Forensic Res 3:158. doi:10.4172/2157-7145.1000158

Copyright: @ 2012 Madadin M, et al. This is an open-access article distributed under the terms of the Creative Commons Attribution License, which permits unrestricted use, distribution, and reproduction in any medium, provided the original author and source are credited. 
while it will be $58 \%$ if taken 72 hour and the IUD If fitted within 5 days, the efficacy will be $99 \%$ [3].

Because the forensic doctors the first doctor usually meet the victim and giving of emergent contraception early is great help and need no skills or specialist to describe it, it is better to be giving by forensic physician rather than refer to Obstetric or ignorance this issue.

\section{HIV Post-Exposure Prophylaxis (PEP)}

Though post exposure prophylaxis has not yet been evaluated following sexual exposure, it has been shown to lower the risk of seroconversion following occupational exposure to HIV by $81 \%$. Victims of sexual assault should be given the best information regarding the importance of post exposure prophylaxis to enable them to make an informed decision [4].

HIV pep given According to the many factors such as Current HIV status and previous exposure to HIV infection, Nature of allegation whether vaginal or anal, use of condom, Time passed since incident and Perpetrator details whether Known to have HIV or not [1].

Efficacy of PEP may be of less value if given after 3 days of the incident, but may be given after this period if the exposure is 'high-risk'. The use of HIV PEP following sexual exposure to HIV is recommended where the victim attends within 3 days of incident.

It may be helpful to use a starter pack (3-5 days medication). Suitable combinations would be Combivirs (zidovudine $300 \mathrm{mg}$ plus lamivudine $150 \mathrm{mg}$ ) bd plus nelfinavir $1250 \mathrm{mg}$ bd. The ideal duration of PEP is unknown. However, animal studies and a case-controlled study of health care workers suggest that a month is required to decrease the transmission of HIV [5].

Again the rule of forensic examiner to evaluate the risk of HIV transmission and also can take action for giving the prophylaxis and further follow up with specialized clinic if needed

\section{Hepatitis PEP}

\section{Indication and role of forensic physician}

There are recommendations of the management and prevention of transmission of Hepatitis by Forensic physician especially type B which has available vaccination and this applied in many countries as UK but unfortunately not applied in many countries which still has population with risk of getting hepatitis $B$ infection.

Sexual transmission of hepatitis B occurs in non immune person ,homosexual and correlates with multiple partners, anal sex and also with oro-anal sex , Transmission also occurs after heterosexual contact e.g. $18 \%$ infection rates for regular partners of patients with acute hepatitis B, Sex workers are also at higher risk [6].

Follow up and screening which usually done in specialized clinic while the role of forensic physician is to Educate the patient about the disease and risk as well as vaccination opportunity, Give vaccine if recommended, Refer to follow up and screening.

Vaccination should be offered to non immune patients in mentioned groups, If a previously non-immune person gives a history of unprotected anal or vaginal sex with a known case or high risk person of hepatitis $B$, post exposure prophylaxis with vaccine should be offered as soon as possible if less than 6 weeks post exposure and screen repeated at 3 months, Hepatitis B specific immunoglobulin should only be given if within 72 hours (up to 1 week) of first exposure with known person of hepatitis B [7].

For Post-exposure prophylaxis, an accelerated schedule of monovalent hepatitis $B$ vaccine (or a combined vaccine of equivalent strength) should be used, with vaccine given at zero, one and two months also commonly given at zero, 7, 21 days [7].

Also can be administered to sexual assault victims at the time of the examination if they have not been previously vaccinated. Follow-up doses of vaccine should be administered 1-2 and 4-6 months after the first dose [8].

\section{STI}

Forensic doctor must consider risk of infectious diseases which may be transmitted during assault, Assessment of risk which depend on assailant condition and nature of sexual assault the forensic doctor action may be either Screening or/and Prophylactic antibiotic.

Screening must be 2-3 weeks post assault and future screening after 3 months to consider incubation period of infection, such screening help in detect and treat infection and reassurance for the victim in addition sometimes has great medico legal importance in link offender to complaint, the appropriate action for forensic physician to refer the victim to specialized infections clinic.

Also sometimes the doctor can give the victim prophylactic antibiotic in cases such as If assailant known to have infection, victim Unable to tolerate screening and Fitting of IUD and this best to be given at time of examination [1].

The Centers for Disease Control and Prevention (CDC) recommends the following Antibiotics Treatment Option

Ceftriaxone (125 mg IM in a single dose)

Metronidazole (2g orally in a single dose)

Azithromycin (1g orally in a single dose) or Doxycycline (100 mg orally twice a day for 7 days) [8].

\section{Emotional Support}

Sexual assault victims often need psychological support. Symptoms like anger, fear, anxiety, pain, sleeplessness, loss of appetite, shame, guilt, depression, and thoughts can appear after days to weeks following the assault. Some victims may not get help because of their fear that disclosure of the incident will be too painful. On the other hand, most victims find counseling helpful for recovery.

In the first few days after an assault, some victims develop physical symptoms, such as pain in the muscles, joints, genitals, pelvis and/or abdomen. They may also suffer from emotional symptoms, such as lack of appetite, difficulty sleeping, or nightmares. Some victims find it very difficult to regain their habits, lifestyles, and sexual relationships. This physical and emotional symptom is called the rape trauma syndrome; which can persist for several months [9].

Several treatment options can assist in coping with the emotional problems accompanying sexual trauma. Early management may help to minimize the risk of long-term issues with depression, anxiety, or posttraumatic stress disorder.

Counseling can be provided by different health workers, including social workers, psychologists, nurses, and psychiatrists. Some victims 
Citation: Madadin M (2012) Medical Aftercare of Sexually Assaulted Victims by Forensic Physician. J Forensic Res 3:158. doi:10.4172/21577145.1000158

prefer to meet with a counselor while others prefer to meet in a group setting with victims who have had similar assaults [10].

\section{Summary and Conclusion}

Patient presenting for care after sexual assault should first be evaluated for acute traumatic physical injuries. Then should be offered medical care, unfortunately this medical care may be missed in some agencies or delayed due to referral to another discipline. Forensic physician must have medical rule not only the legal, he must provide the optimum care he can give and refer to specialized clinic to continue the care of the victim.

We suggest as many countries are doing in this field, the forensic physician (or team) is responsible for providing this medical care and prophylaxis then refer if needed, such protocol has many advantages rather than only legal rule of forensic doctor as only document the injuries and refer the victim to complete her medical care or sometimes neglect such refer and ignorance the harm on the victim. This care includes prophylaxis for sexually transmitted infections and pregnancy finally, safe plans for discharge, including planned follow-up for medical care and psychological support, are critical.

Many countries has such rule and they have guidelines and recommendation, in other hand we would like to introduce such rule of forensic doctor in our Arab countries and protocol must be established to minimize the physical and psychological trauma to the victim

\section{Acknowledgement}

We would like to thank St. Marry centre, Manchester, UK, to accommodate us and looking for how such care provided optimally, we would like to thank the whole team specially Dr. Catherine White, Mrs. Bernie Ryan.

\section{References}

1. http://www.medicines.org.uk/emc/

2. Faculty of sexual \& reproductive healthcare clinical guidance ,Intrauterine Contraception, November 2007.

3. National guideline for the management of adult victims of sexual assault Clinical Effectiveness Group (Association of Genitourinary Medicine and the Medical Society for the Study of Venereal Diseases). Sex Transm Infect 1 : S82-S84.

4. Bamberger JD, Waldo CR, Gerberding JL, Katz MH (1999) Postexposure prophylaxis for human immunodeficiency virus (HIV) infection following sexual assault. Am J Med 106: 323-326.

5. Fisher M, Benn P, Evans B, Pozniak A, Jones M, et al. (2006) UK Guideline for the use of post-exposure prophylaxis for HIV following sexual exposure. Int J STD AIDS 17: 81-92.

6. United Kingdom national guideline on the management of the viral hepatitides A, B \& C 2008 (2008). London (UK): British Association for Sexual Health and HIV (BASHH): 27.

7. http://www.dh.gov.uk/health/category/publications/reports-publications/

8. Workowski KA, Berman S; Centers for Disease Control and Prevention (CDC) (2010) Sexually transmitted diseases treatment guidelines, 2010. MMWR Recomm Rep 59: 1-110.

9. Kimerling R, Calhoun KS (1994) Somatic symptoms, social support, and treatment seeking among sexual assault victims. J Consult Clin Psychol 62 333-340.

10. Bates CK (2012) Patient information: Care after sexual assault (Beyond the Basics). 